\title{
Effect of Probiotics on Infantile Colic in Breast-Fed Infants: A Randomized Single-Blind Clinical Trial
}

\author{
Hamed Roohanizadeh ${ }^{1}$, Hasan Karami ${ }^{1}$ and Seyyed Abbas Hashemi ${ }^{2, *}$ \\ ${ }^{1}$ Departments of Pediatrics, Mazandaran University of Medical Sciences, Sari, Iran \\ ${ }^{2}$ Department of Internal Medicine, Mazandaran University of Medical Sciences, Sari, Iran
}

\begin{abstract}
Aim: This study examined the impact of probiotics on children's colic in breast-fed infants.
Methods: This study was a randomized single-blind clinical trial. 100 infants were referred to pediatric gastroenterology clinic of Avicenna diagnosed by gastroenterologist of children who suffered from infantile colic and they were qualified after justifying parents and obtaining written consent. (IRCT registration number: IRCT2016082829573N1).

Results: Results showed that there was a statistically significant difference in terms of crying time $(p=0.000)$ and the arrival and departure of the group. $(p=0.000)$.

Conclusion: The outcomes indicated that using probiotics could reduce colic in infants and improve the quality of life in this group.
\end{abstract}

Keywords: Probiotics, colic, infantile.

\section{INTRODUCTION}

Infantile colic is due to situations like anger and abundant in many hours of crying on consecutive days ( 3 hours per day or more than 3 hours of continuous crying, 3 or more than 3 days per week) $[1,2]$ in the first months of life causing serious concern and families suffer that impose unnecessary costs, including visiting several doctors, clinical measures and using multiple and various drugs.

On the other hand, infantile colic is very common (10 to $30 \%)$ and despite numerous investigations have been conducted, there is no cure for this disease [2-7], which causes anxiety, confusion of family and spending time of many physicians to justify families. According to the above issues to find a way to treat the disease appears to be necessary.

Acute and unexpected abdominal pain is common. In children, a syndrome characterized by self-limiting behaviors in the first 3 months of secondary life to a specific underlying gastrointestinal disorders and bowel symptom is seen. Of course, neither the origin nor painful gastrointestinal pain, it has not been proven.

Most infants with this syndrome are normal in other situation. Colic in 10 to $25 \%$ of cases occurred in $30 \%$ of cases, symptoms may continue for 4 and 5 months. Behavioral characteristics of colic syndrome are:

*Address correspondence to this author at the Department of Internal Medicine, Mazandaran University of Medical Sciences, Sari, Iran;

E-mail: Abbas.hashemi30@gmail.com increasing daily crying time in the first 2 months and then decreased crying at 3 th and $4^{\text {th }}$ month, increased crying in the late afternoon and evening, prolonged crying episodes with some patients resistant to calm down, close the fist and bending the legs on abdomen, backache, redness of the face and skin, face pain, abdominal and rigid distension, regurgitation and flatus and begin and ending abruptly, which can be unrelated to environmental issues $[8,9]$.

\section{Prebiotics}

Prebiotic include substances that reinforce the proliferation of probiotic microorganisms including fructose and galactose, oligosaccharides, insulin and foods made with rye and Psylum that are germinated.

It is necessary that prebiotics pass unharmed selective substrates from the upper gastrointestinal tract in the large intestine [10]. Some specific types of probiotics may be preventive or have therapeutic effects on acute infectious diarrhea in children and reduce the amount and duration of it.

Beneficial effect of probiotics in infectious diarrhea in adults has been shown [11]. Prophylactic prescription of oral probiotics reduces the incidence and severity of NEC [12]. Lactase deficiency: lactase deficiency is a very common phenomenon in children.

Gastrointestinal side effects are observed after consumption of milk and dairy products. These effects include bloating, diarrhea and abdominal distension. Probiotic bacteria are able to digest lactose and thus 
can eliminate symptoms of lactase deficiency in susceptible individuals.

In fact, children with a deficiency of yogurt lactase (not milk) containing the probiotic bacteria that can destroy the symptoms and reduce the concentration of exhaled hydrogen $[13,14,15]$. According to research in the treatment of infantile colic (in various forms) by probiotics has various results [2-7] and it seems that more research is needed. For this reason, this study examines the impact of probiotics on children's colic in breast-fed infants.

\section{MATERIALS AND METHODS}

This study was a randomized single-blind clinical trial (IRCT registration number: IRCT2016082829573N1) 100 infants were referred to pediatric gastroenterology clinic of Avicenna diagnosed by gastroenterologist of children who suffered from infantile colic and they were qualified after justifying parents and obtaining written consent.

1. Age between 15 and 120 days

2. Mother milk consumption

3. Knot use of antibiotics

4. Time of continuous crying for 3 hours or more than 3 hours per day, 3 days or more than 3 days a week

5. Birth weight between 2,500 and 4,000 grams

If you have any of the following conditions during the study, these are excluded at the beginning until examination.

1. Start formula

2. The reasons which cause antibiotics application

3. Identify the cause of pain in infants, such as Gastro Esophageal Reflux and... (Introduction of organic causes of abdominal pain)

4. Unwillingness of parents to continue studying

5. Existence of underlying disease

6. Any illness in patients diagnosed at the age of 6 months, Which would imitate the symptoms of infantile colic.

Patients were randomly divided into two groups of 50 persons. The control group was given a placebo and the case group was given Biogaia probiotic drops (containing Lactobacillus router manufactured by Ferring Company in Germany and company of Dayatb in Iran) prescribed orally for 28 days.

Medication drop in the volume of $5 \mathrm{cc}$ (containing Lactobacillus router was 108 in 5 drops) prescribed daily at a rate of 5 drops. Placebo drops were provided quite similar to drop drug (in terms of shape, size, color).

Assuring parents, embracing and proper feeding methods are identical in both groups and were instructed to parents at the beginning of training as follows:

1. Record the exact start time and end of the day crying and crying all the time in hours and minutes during a month, the number of colic attacks per day

2. The correct method of breast-feeding, "according to the baby desire and burping the baby was taken after each feeding.

3. excluding other causes of baby crying, mother was instructed in case of any changes in the patient's clinical course of disease apart from the diagnosis of colic (include fever, runny nose, cough, avoid milk, lethargy, severe cases, discoloration of urine, dysuria,...) to see the doctor again if the diagnosis is not colic, child is excluded and received relevant treatment relating to disease.

Tracking the results was associated with clinical evaluation on the first day, the seventh day, $14^{\text {th }}, 21$ th and $28^{\text {th }}$ days as weekly dose of probiotic drops of Biogaia (which is not less than 10 and timetables of baby crying were recorded per day by mother as a weekly average, evaluated by a statistician. Also, the infant was examined from 6 months of his life to explore other possible diseases that were not seen at the beginning of the study.

According to reference [4], by $95 \%$ of confidence level and power of $90 \%$ of volume equal to sample consisted of 36 persons for each group that was selected due to the loss of 10 percent, 50 patients for each group were selected. To describe data, the frequency tables, diagrams, mean index and the standard deviation was applied for the comparison between the two groups with regard to the repeated measure, analysis of variance with repeated measures 
was used to compare the groups in terms of confounding variables, T-test, and $x 2$ test used in case of significant differences based on regression techniques.

\section{RESULTS}

The results of this study were a randomized singleblind as a clinical trial. 100 infants referred to Avicenna pediatric gastroenterology clinic diagnosed by gastroenterologist of children with infantile colic randomly and divided into two groups and their demographic characteristics, the age of pregnancy, parental smoking history, history of dairy, stool consistency and reduce crying in both groups were recorded. Patients' demographic data in Table $\mathbf{1}$ is expressed. Statistical analysis was carried out between the two groups. There was no significant difference in any of the following factors.

Following the results, baby crying was recorded by mother on the first day, first week, second week, third week and the fourth week as shown in Table 2.
Statistical analysis was carried out between the two groups, had a statistically significant difference in terms of crying time $(p=0.000)$.

The study was carried out within the group between the arrival and departure of the group that was under treatment with a significant difference $(p=0.000)$.

\section{DISCUSSION AND CONCLUSION}

Infantile colic in the first months of life caused great concern, suffering families and imposing unnecessary costs, including visiting several doctors, clinical measures, multiple and various drugs.

On the other hand, infantile colic is very common (10 to $30 \%$ ) [16] And many types of research has done despite numerous investigations, there is no definitive cure for this disease that causes anxiety and confusion of his family and spends a lot of time to justify the family.

Based on the above issues, to find a way to treat the disease appears to be necessary.

Table 1:

\begin{tabular}{|c|c|c|c|c|}
\hline & & Case N(\%) & Control N(\%) & $P$ value \\
\hline \multirow{2}{*}{ gender } & Male & (48)24 & $(50) 25$ & \multirow{2}{*}{0.686} \\
\hline & female & (52)26 & $(50) 25$ & \\
\hline \multirow{2}{*}{ Method of delivery } & Caesarean & $(88) 44$ & $(74) 37$ & \multirow{2}{*}{0.118} \\
\hline & Normal delivery & $(12) 6$ & (26)13 & \\
\hline \multirow{3}{*}{ Gestational age } & preterm & (26)13 & (36)18 & \multirow{3}{*}{0.385} \\
\hline & term & $(68) 34$ & $(62) 31$ & \\
\hline & Post term & (4)2 & (2)1 & \\
\hline \multirow{3}{*}{ Form of defecation } & watery & (38)19 & (42)21 & \multirow{3}{*}{0.848} \\
\hline & smooth & (44)22 & (46)23 & \\
\hline & firm & $(16) 8$ & $(12) 6$ & \\
\hline Dairy in parents & & $(8) 4$ & $(8) 4$ & 0.187 \\
\hline Smoke in parents & & (4)2 & $(8) 4$ & 0.9 \\
\hline
\end{tabular}

Table 2: Crying Time Based on Weeks

\begin{tabular}{|c|c|c|c|}
\hline & Case & Control & P Value \\
\hline \hline Day 1 & $5 / 1 \pm 85 / 3$ & $8 / 1 \pm 1 / 5$ & 0.6 \\
\hline Week 1 & $4 / 9 \pm 3 / 22$ & $1 / 9 \pm 4 / 33$ & 0.000 \\
\hline Week 2 & $6 / 10 \pm 9 / 16$ & $03 / 11 \pm 9 / 30$ & 0.000 \\
\hline Week 3 & $9 / 13 \pm 6 / 11$ & $8 / 11 \pm 9 / 24$ & 0.000 \\
\hline Week 4 & $5 / 11 \pm 2 / 9$ & $5 / 13 \pm 6 / 24$ & 0.000 \\
\hline
\end{tabular}


In this randomized study as a single-blind clinical trial, 100 infants were divided into two groups of 50 persons as a control group and receiving probiotic to examine the effect of probiotics on infantile colic in $\mathrm{Bu}-$ Ali Sina clinic as infant gastro-intestinal Hospital.

The results of this study showed that, for the first day of drug prescription, in the $7^{\text {th }}, 14^{\text {th }}, 21^{\text {th }}$, and $28^{\text {th }}$ days in the survey, statistically significant differences are seen between the two groups. The probiotic drug significantly reduced the time crying and irritability in patients. A study in 2012 by Valerie Sung and colleagues [17] in Australia was performed to investigate the effect of probiotics in the treatment of infantile colic. In their study, 160 infants less than 3 months were evaluated.

Similar to our study, they also study these individuals on $7^{\text {th }}, 14^{\text {th }}, 21^{\text {th }}$, and $28^{\text {th }}$ days. Results of the study in accordance with the present study showed that using probiotics reduce irritation and reduce colic in babies.

In a study done by $F$ Savino and colleagues [16] in Italy in 2007, 90 breastfed colicky infants were selected to receive probiotics for 28 days.

Parents and children can record their daily crying time and side effects by questionnaire. Among the 83 infants who completed the study, 41 patients were included in the probiotic group and 42 patients were included in the simethicone group. $\mathrm{P}<0.001$.

In the seventh day, average daily crying time in the probiotic group was 59 minutes per day, in Simethicone group was 177 minutes per day and in $28^{\text {th }}$ day, in the probiotic group was 51 minutes per day, and in the simethicone group was 145 minutes per day ( $P$ $<0.001)$.

In $28^{\text {th }}$ day, 39 patients (95\%) had responded to probiotics while 3 patients had responded to simethicone and side effects were not observed. This study showed that the treatment with probiotics for a week will improve colic in breastfed infants [4].

As a result, the current study also showed that the use of probiotics improve colic and reduce crying in infants.

Jose M Saavedra and colleagues [13] in a study carried out in America in 2004, he examined the effect of milk containing probiotic as a prospective, doubleblind, randomized and placebo-controlled study in the infantile period of 3 to 24 months. 118 infants had received formula during $127+-210$ days. Two groups didn't indicate a significant difference in terms of age, sex, prescription of formula. This formula containing supplement was confirmed. In this group, few cases of colic or irritability (P:0.001) and antibiotics (P:0.001) compared to non-supplemented formula were reported.

In terms of growth, referring to the hospital and daily problems, the difference between the two groups is not observed. As a result of this research, the current study also showed that long-term consumption of probiotic milk is well tolerated, safe, causes adequate growth, reduce colic, irritability, and lower consumption of antibiotics.

\section{CONCLUSION}

Intestinal surface creates a wide area of contact between the inside and outside of the human body. The results of several studies have shown that microbes play an important role in the evolution of the immune system, digestive system, and the central nervous system.

Manipulation of microbes in many cases can be the originator of the desired results in the human body. The results showed that using probiotics could reduce colic in infants and improve the quality of life in this group. Although further studies on larger numbers of patients are needed to confirm these results.

\section{REFERENCES}

[1] Steutel NF, Benninga MA, Langendam MW, de Kruijff I, Tabbers MM. Reporting Outcome Measures in Trials of Infant Colic: A Systematic Review. Journal of Pediatric Gastroenterology and Nutrition 2014. https://doi.org/10.1097/MPG.0000000000000412

[2] Savino F, Tarasco V, Sorrenti M, Lingua C, Moja L, Gordon $M$, et al. Dietary modifications for infantile colic. The Cochrane Library 2014. https://doi.org/10.1002/14651858.CD011029

[3] Savino F, Cresi F, Pautasso S, Palumeri E, Tullio V, Roana $\mathrm{J}$, et al. Intestinal microflora in breastfed colicky and non-colicky infants. Acta Paediatrica 2004; 93: 825-829. https://doi.org/10.1111/j.1651-2227.2004.tb03025.x

[4] Savino F, Pelle E, Palumeri E, Oggero R, Miniero R. Lactobacillus reuteri (American Type Culture Collection Strain 55730) versus simethicone in the treatment of infantile colic: a prospective randomized study. Pediatrics 2007; 119: e124-e130.

[5] Saavedra JM, Abi-Hanna A, Moore N, Yolken RH. Long-term consumption of infant formulas containing live probiotic bacteria: tolerance and safety. The American Journal of Clinical Nutrition 2004; 79: 261-267. https://doi.org/10.1093/ajcn/79.2.261

[6] Rosen LD. "The Gripe": An Integrative Approach to Infant Colic. Explore: The Journal of Science and Healing 2007; 3 : 417-422. https://doi.org/10.1016/j.explore.2007.05.013 
[7] Connolly E, Mollstam B. Use of selected lactic acid bacteria for reducing infantile colic. In: Google Patents; 2007.

[8] Barr RG, Rotman A, Yaremko J, Leduc D, Francoeur TE. The crying of infants with colic: a controlled empirical description. Pediatrics 1992; 90: 14-21.

[9] Brazelton TB. Crying and colic. Helping Parents Solve Their Children's Behavior Problems 1998; 239.

[10] Schrezenmeir J, de Vrese M. Probiotics, prebiotics, and synbiotics-approaching a definition. The American journal of clinical nutrition 2001; 73: 361s-364s. https://doi.org/10.1093/ajen/73.2.361s

[11] Ouwehand AC, Derrien M, de Vos W, Tiihonen K, Rautonen $\mathrm{N}$. Prebiotics and other microbial substrates for gut functionality. Current Opinion in Biotechnology 2005; 16: 212-217. https://doi.org/10.1016/j.copbio.2005.01.007

[12] Preidis GA, Versalovic J. Targeting the human microbiome with antibiotics, probiotics, and prebiotics: gastroenterology enters the metagenomics era. Gastroenterology 2009; 136: 2015-2031.

https://doi.org/10.1053/j.gastro.2009.01.072
[13] Saavedra J, Tschernia A. Human studies with probiotics and prebiotics: clinical implications. British Journal of Nutrition 2002; 87: S241-S246. https://doi.org/10.1079/BJN/2002543

[14] Andersson H, Asp N-G, Bruce A, Roos S, Wadström T, Wold AE. Health effects of probiotics and prebiotics. A literature review on human studies. Food \& Nutrition Research 2001; 45: 58-75.

[15] Savino F, Bailo E, Oggero R, Tullio V, Roana J, Carlone N, et al. Bacterial counts of intestinal Lactobacillus species in infants with colic. Pediatric Allergy and Immunology 2005; 16: 72-75.

https://doi.org/10.1111/j.1399-3038.2005.00207.x

[16] Savino F. Focus on infantile colic. Acta Paediatrica 2007; 96 : 1259-1264.

https://doi.org/10.1111/j.1651-2227.2007.00428.x

[17] Sung V, Hiscock $H$, Tang M, Mensah FK, Heine RG, Stock A et al. Probiotics to improve outcomes of colic in the community: Protocol for the Baby Biotics randomised controlled trial. BMC Pediatrics 2012; 12: 135. https://doi.org/10.1186/1471-2431-12-135 